\title{
Theoretical and Experimental Investigation Into the Influence Factors for Range Gated Reconstruction
}

\author{
Sing Yee CHUA ${ }^{1}$, Xin $\mathrm{WANG}^{1 *}$, Ningqun $\mathrm{GUO}^{1}$, and Ching Seong TAN ${ }^{2}$ \\ ${ }^{1}$ School of Engineering, Monash University Malaysia, Jalan Lagoon Selantan, Bandar Sunway, 47500 Selangor, \\ Malaysia \\ ${ }^{2}$ Faculty of Engineering, Multimedia University, Jalan Multimedia, 63000 Cyberjaya, Selangor, Malaysia \\ *Corresponding author: Xin WANGＥ-mail: wang.xin@monash.edu
}

\begin{abstract}
Range gated is a laser ranging technique that has been applied in various fields due to its good application prospects. In order to improve the effectiveness of this method, influence factors contributing to the system performance should be well understood. Thus this paper performs theoretical and experimental investigation to comprehend the effects caused by multiple factors on range gated reconstruction. Our study focuses on the distance, target reflection, and acquisition time step parameter where their impacts on the quality of range reconstruction are analyzed. The presented experimental results show the expected trends of range error to support the validity of our theoretical model and discussion which can be used in future improvement works.
\end{abstract}

Keywords: Laser ranging; reflection; sensor

Citation: Sing Yee CHUA, Xin WANG, Ningqun GUO, and Ching Seong TAN, "Theoretical and Experimental Investigation Into the Influence Factors for Range Gated Reconstruction," Photonic Sensors, 2016, 6(4): 359-365.

\section{Introduction}

Over the past decades, laser ranging has been a popular approach in optical metrology because of its unique characteristics of non-contact and nondestructive nature [1]. As of today, laser ranging has been applied in various fields such as oceanic and environmental research, surveillance, industry, and day-to-day applications [2].

Range gated is a laser ranging technique operates based on time-of-flight (TOF) principle to measure the travel time between the emitted laser pulse and the pulse reflected from the target. Pulsed laser and sensor's gate are controlled simultaneously to capture the reflected pulse where range $r$ is determined from the round trip time $t$ and the speed of light $c$.

$$
r=\frac{c t}{2} .
$$

Range gated has been a promising method in applications such as target detection and recognition [3], night vision $[4,5]$, underwater $[6,7]$, and three-dimensional (3D) imaging [8, 9]. Besides, continuous development in laser, sensor, signal processing, and computer technology further improves the cost effectiveness of this approach. The good application prospects motivate the study into the influence factors for range gated reconstruction which can contribute to improve the system performance.

In a range gated system, laser pulse interacts with the target surface to generate a backscatter signal which contains the key information for range reconstruction. Hence, the quality of range 
reconstruction strongly relies on the reflected laser pulse from the target which undergoes changes along the propagation. Essentially, the detected laser pulse is affected by the laser source, sensor, target, and atmospheric effect [11]. These parameters could change the characteristics and cause variations in the reflected laser pulse which directly impact the accuracy of range determination. The importance of laser intensity profile [11, 12], distance interference [10], sensor [13, 14], and scattering effects [16] were discussed in various literatures.

In this paper, range gated reconstruction is analyzed theoretically and experimentally to obtain a comprehensive understanding and relationship between the influence factors and ranging performance. In Section 2, a brief of range gated technique is given, and theoretical derivation and analysis of 3D range gated reconstruction model are presented. On the other hand, the experimental setup for our investigation is described in Section 3. The impact of multiple influence factors is analyzed and discussed in Section 4. Finally, a conclusion is given in Section 5.

\section{Theoretical derivation of range gated reconstruction model}

Range gated approach operates based on TOF concept by measuring the round trip time between the emitted laser pulse and the pulse echo resulting from its reflectance off the target. The working principle of a range gated imaging system using time slicing technique is illustrated in Fig. 1. Pulsed laser is used as the illumination source, and gated camera is time delayed to open only for a very short duration normally in nanoseconds or picoseconds to capture the reflected image slice from the target over a distance. Synchronization between the laser and gated camera is particularly important. Camera gate remains closed when the laser pulse is emitted towards target. Camera gate is configured to open at the designated delayed time to capture the visible time slice reflected in the form of intensity image.

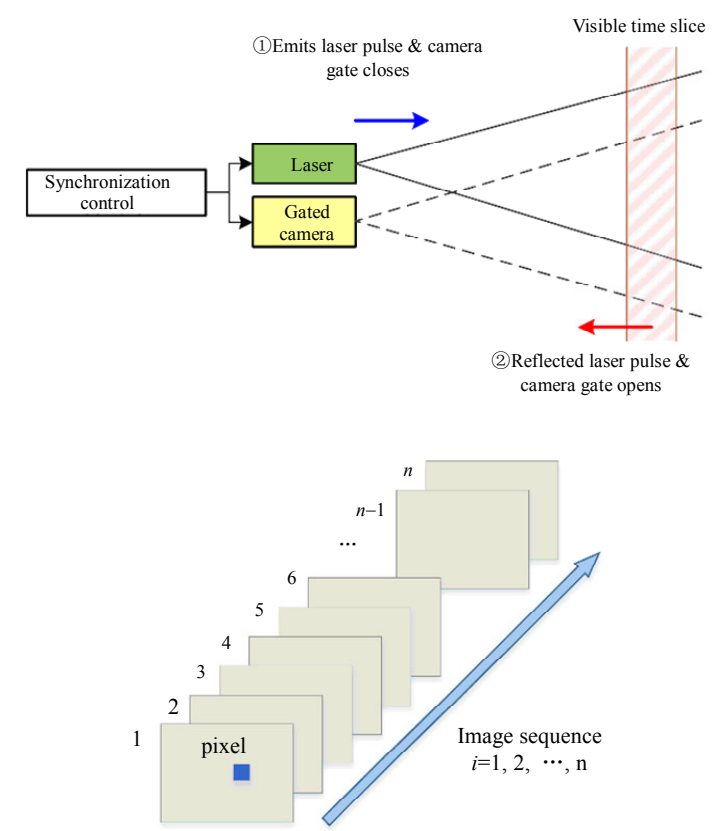

Fig. 1 Operating principle of range gated imaging system.

Based on time slicing technique $[8,9,12]$, the camera gate $G(t)$ is delayed by time $t_{i}$ with a time step $t_{\text {step }}$ to acquire an image sequence $i=1,2,3, \cdots, n$. Intensity captured in an image pixel $I_{i}(x, y)$ is the incident energy of reflected laser pulse $P_{r}(t)$ integrated when the camera gate is opened for a $t_{\text {gate }}$ time which can be expressed as

$$
I_{i}(x, y)=\int P_{r}\left(t-\frac{2 r}{c}\right) G\left(t-t_{i}\right) d t .
$$

Typically, the camera gate $G(t)$ is assumed as constant; hence the pixel intensity relies on the reflected laser energy $P_{r}(t)$. From laser detection and ranging (LADAR) range equation, the received signal $P_{r}$ is defined as [17]

$$
P_{r}=\frac{\eta_{\mathrm{sys}} \eta_{\mathrm{atm}} D^{2} \rho A P_{t}}{r^{2} \theta_{R}\left(\theta_{t} r\right)^{2}}
$$

where $P_{r}$ and $P_{t}$ are the received and transmitted signals across range $r$, and $\eta_{\text {sys }}$ and $\eta_{\text {atm }}$ represent the system efficiency factor and atmospheric transmission loss caused by absorption and scattering. $D$ is the diameter of receiver aperture, and $\rho$ is the target surface reflectivity. $\theta_{t}$ represents the laser transmitter beam diameter and angular divergence, and $\theta_{R}$ is the solid angle over which radiation is dispersed upon reflection. 
Assume the target surface area $A$ is equal to the projected area of laser beam [17]

$$
A=\frac{\pi \theta_{t}^{2} r^{2}}{4} \text {. }
$$

Equation (3) can be simplified as

$$
P_{r}=\frac{\pi \eta_{\mathrm{sys}} \eta_{\mathrm{atm}} D^{2}}{4 r^{2}} \frac{\rho}{\theta_{R}} P_{t}
$$

where $\rho / \theta_{R}$ corresponds to the target reflection characteristics which we can represent with a bidirectional reflection distribution function (BRDF) model [18] where $K_{S}$ and $K_{D}$ are the specular and diffuse reflection constants, $\theta$ is the angle of incidence and reflection, $s$ is the surface slope, and $m$ is the diffusivity coefficient.

$$
\mathrm{BRDF}=\frac{K_{S}}{\cos ^{6} \theta} \exp \left(\frac{-\tan ^{2} \theta}{s^{2}}\right)+K_{D} \cos ^{m} \theta .
$$

Gaussian form is commonly assumed for temporal function of the transmitted laser pulse $P_{t}(t)$ where $P_{o}$ represents the transmitted power and $\sigma_{p}$ denotes the standard deviation of laser pulse $[9,13]$ :

$$
P_{t}(t)=\frac{P_{o}}{\sqrt{2 \pi} \sigma_{p}} \exp \left(\frac{-t^{2}}{2 \sigma_{p}^{2}}\right) .
$$

Accordingly, (5) can be written as

$$
\begin{aligned}
P_{r}(t)= & \frac{\pi \eta_{\mathrm{sys}} \eta_{\mathrm{atm}} D^{2}}{4 r^{2}} \frac{P_{o}}{\sqrt{2 \pi} \sigma_{p}} \exp \left(\frac{-t^{2}}{2 \sigma_{p}^{2}}\right) . \\
& {\left[\frac{K_{S}}{\cos ^{6} \theta} \exp \left(\frac{-\tan ^{2} \theta}{s^{2}}\right)+K_{D} \cos ^{m} \theta\right] . }
\end{aligned}
$$

Using time slicing technique, the summation of radiant energy in the image pixel can be seen as the integration over time slices $\int d t_{i} / t_{\text {step }}$ as the time step for image acquisition is much smaller than the laser pulse width and camera gate [9]:

$$
I(x, y)=\sum_{i} I_{i}(x, y)=\frac{\int I_{i}(x, y) d t_{i}}{t_{\text {step }}} .
$$

Based on (2), we can further simplify $I(x, y)$ as

$$
I(x, y)=\frac{\int P_{r}(t) d t \int G(\tau) d \tau}{t_{\text {step }}} .
$$

By substituting $P_{r}(t)$ from (8) into (10) and assume
$G(\tau)=1$ when $0 \leq \tau \leq t_{\text {gate }}, I(x, y)$ becomes

$$
\begin{aligned}
I(x, y)= & \frac{\pi \eta_{\text {sys }} \eta_{\text {atm }} D^{2}}{4 r^{2} t_{\text {step }}} . \\
& {\left[\frac{K_{S}}{\cos ^{6} \theta} \exp \left(\frac{-\tan ^{2} \theta}{s^{2}}\right)+K_{D} \cos ^{m} \theta\right] . } \\
& \frac{P_{o}}{\sqrt{2 \pi} \sigma_{p}} \int_{-\infty}^{\infty} \exp \left(\frac{-t^{2}}{2 \sigma_{p}^{2}}\right) d t \int_{0}^{t_{\text {gate }}} d \tau .
\end{aligned}
$$

This equation eventually resolved into

$$
\begin{aligned}
I(x, y)= & \frac{\pi \eta_{\text {sys }} \eta_{\text {atm }} D^{2}}{4 r^{2}} P_{o} \frac{t_{\text {gate }}}{t_{\text {step }}} . \\
& {\left[\frac{K_{S}}{\cos ^{6} \theta} \exp \left(\frac{-\tan ^{2} \theta}{s^{2}}\right)+K_{D} \cos ^{m} \theta\right] . }
\end{aligned}
$$

Signal to noise ratio (SNR) is an important parameter in analyzing the system performance [19]. SNR is defined as the ratio between the reflected intensity and the associated noises. For our range gated reconstruction, SNR can be expressed as follows after substitute $\sum_{i} I_{i}$ from (9) [10]:

$$
\mathrm{SNR}=\frac{\sum_{i} I_{i}}{\sqrt{\sum_{i}\left(\delta I_{i}\right)^{2}}}=\frac{\int I_{i} d t_{i}}{t_{\text {step }} \sqrt{\sum_{i}\left(\delta I_{i}\right)^{2}}} .
$$

Uncertainty in the two-way travel time is given by the acquisition time step $t_{\text {step }}$; hence the expected range error can be written as

$$
\delta r=\frac{c}{2} t_{\text {step }}=\frac{c}{2} \frac{\int I_{i} d t_{i}}{\mathrm{SNR} \sqrt{\sum_{i}\left(\delta I_{\mathrm{i}}\right)^{2}}} .
$$

Average range $\langle r\rangle$ and two-way travel time $<t>$ of an image pixel $(x, y)$ can be determined from the captured intensity over an image sequence $i=1,2$, $3, \cdots, n$ using weighted average method:

$$
<r>(x, y)=\frac{c<t>}{2}=\frac{c}{2} \frac{\sum_{i=1}^{n} I_{i}(x, y) t_{i}}{\sum_{i=1}^{n} I_{i}(x, y)} .
$$

The calculated range $<r>$ strongly relies on the reflected intensity which is influenced by various factors as shown in (12). In addition, range accuracy is impacted by SNR which is proportional to the 
reflected intensity when the system noise level remains unchanged. Generally, the decreased SNR results in higher range errors. Based on the range gated reconstruction model derived, the relationship between the reflected intensity $I$ and various influence factors in the system is shown as well as their impact to the SNR and range error. Experimental study involves a few factors including the distance, target reflection, and acquisition time step to validate our theoretical discussion.

\section{Experimental setup}

In order to investigate the effects induced by various influence factors, an experimental setup as illustrated in Fig. 2 is used. A pulsed diode pumped solid state Q-switched Nd:YAG laser that operates at wavelength $532 \mathrm{~nm}$ with output energy up to $1 \mathrm{~mJ}$ is used. Silicon high speed biased non-amplified photodetector with active diameter of $400 \mu \mathrm{m}$ and $<300 \mathrm{ps}$ rise/fall time is used to detect the laser pulses in the emitting or reflecting direction. Photodetector transforms the optical pulse into the usable signal for analysis via oscilloscope.

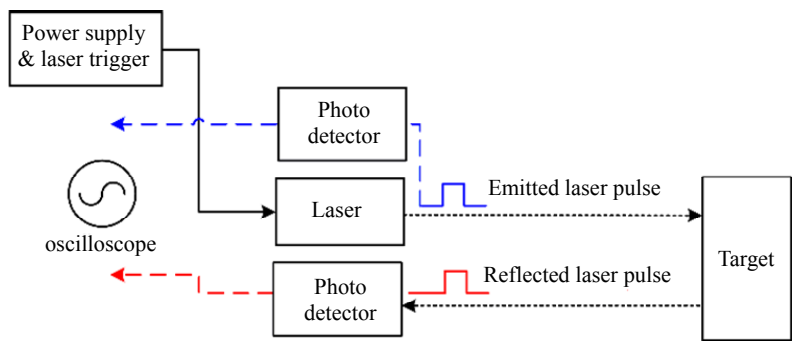

Fig. 2 Schematic diagram of experimental setup to capture the emitted and reflected laser pulse for investigation.

A backscatter signal is produced after the emitted laser pulse interacts with the target surface and is received by the detector in the form of time function. Two-way travel time across the distance between target and the detector is determined from the time difference between the emitted and reflected laser pulse. Correspondingly, the distance or range $r$ can be obtained based on the TOF principle. For our study, the experiment is designed to focus on three factors: distance, target reflection, and acquisition time step where the reflected intensity and range error are analyzed.

\section{Analysis of influencing factors}

\subsection{Distance}

The reflected intensity model derived as (12) shows that the reflected laser energy underlies an inverse range-squared dependency. Using the experimental setup described in Section 3, variation of the reflected intensity across distance is studied. The analyzed results are summarized in Fig. 3 where it clearly shows an inverse range-squared relationship of the reflected intensity [10].

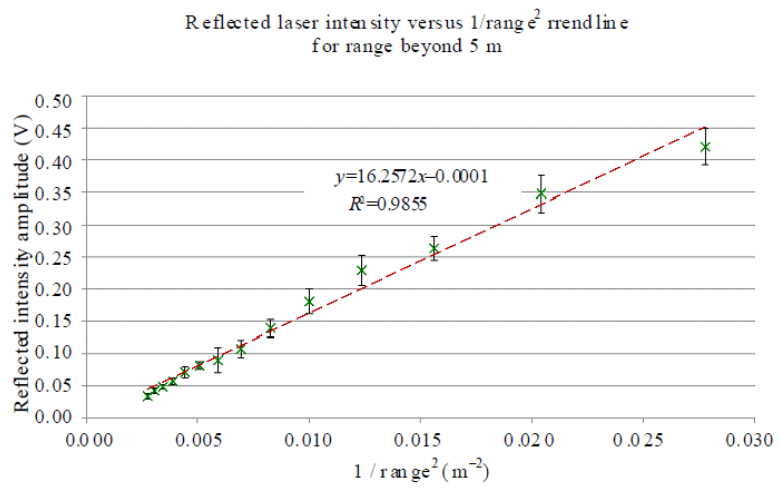

Fig. 3 Measured reflected laser intensity versus $1 /$ range $^{2}$ trendline [10].

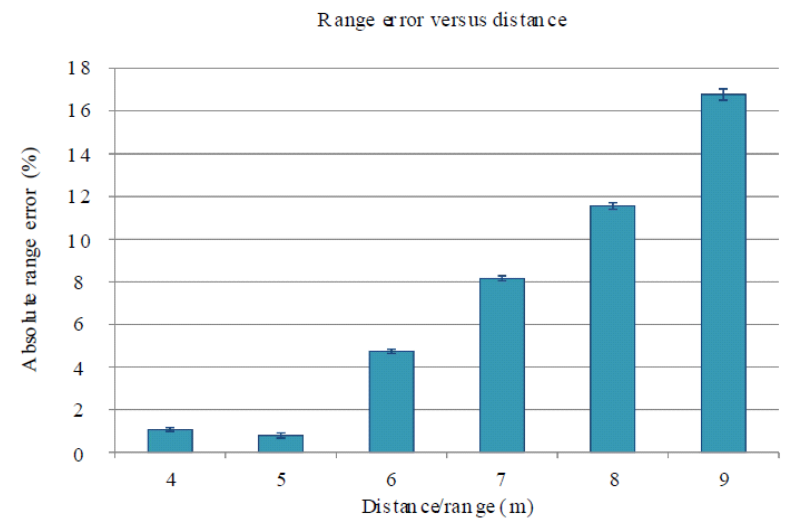

Fig. 4 Comparison of range error versus distance/range under the same constant setup condition.

Because of the reduced intensity over distance, SNR decreases, and we expect higher range error as deduced from (14). Figure 4 shows the range error calculated using weighted average method based on 
30 measurements captured at different distances. The data sets are acquired under the same setup condition to ensure the range error is not influenced by other parameters in the system. The results show that an increase in distance causes a proportional decrease in the reflected intensity and leads to increasing range error as observed which agrees well with our theoretical discussion.

\subsection{Target reflection}

Reflected intensity strongly depends on the characteristics of the target surface [20]. Although Lambertian target (ideal diffuse surface) is commonly assumed due to its simplicity, target reflection is in fact far more complicated, and BRDF concept is normally used to describe that. Our theoretical model has adopted a BRDF model given by (6) which consists of specular and diffuse reflection to analyze the characteristics of reflected intensity in this study. Reflection off a rough surface returned in many directions leads to diffuse reflection while reflection from a smooth surface remains concentrated with the angle of reflection which causes specular reflection. Any target surface practically exhibits mixture of specular and diffuse behavior per surface properties such as roughness and absorption level.

Simulation based on the BRDF model is shown in Fig. 5 where four examples of target surface model are compared. These include two extreme cases of pure specular and pure diffuse surface models, and two examples of mixed components surface with different ratios of surface glint to diffuse behaviour given by specular and diffuse reflection constants, i.e. $K_{S} / K_{D}$. The amplitude of the reflection is maximum when angle of incidence $\theta=0$ degree and decreases when $\theta$ increases, adheres to the BRDF model. As a result, the decreased intensity causes the reduced SNR which gives rise to range error.

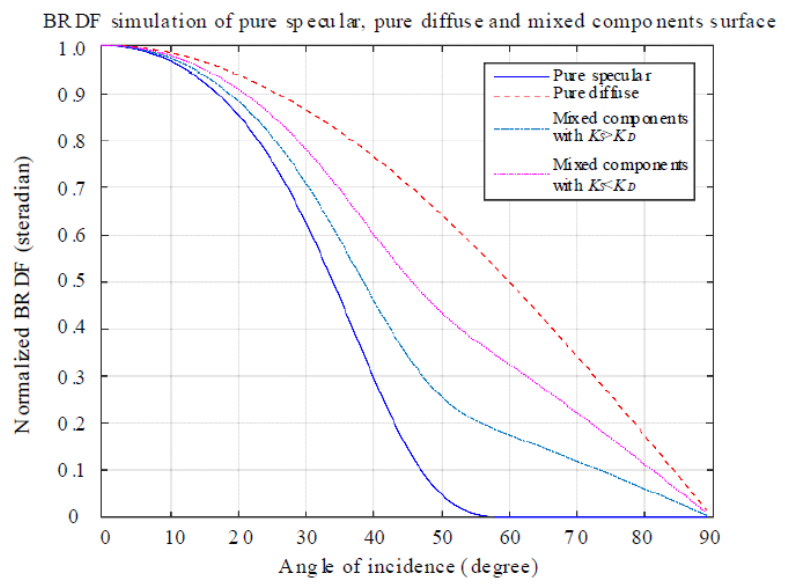

Fig. 5 BRDF simulation as a comparison of different target surface models.

For our experimental study, various target surface materials and roughnesses are tested. Figure 6 compares the range error for target surfaces captured at $5 \mathrm{~m}$, and the results are analyzed based on average of 30 measurements. From the results, we observe that the range error is higher for rough and weak reflective surfaces as compared to smooth and strong reflective surfaces [21] where these surfaces can be modeled using BRDF described in our theoretical model. In addition, the effect of angle variation is evaluated for various target surfaces where the corresponding range error is shown in Fig. 7. It can be clearly seen that the range error is minimum at zero angle of incidence $\theta=0$ degree and increases with the angle of incidence in general. This has demonstrated the angular dependency which agrees with the theoretical model discussed.

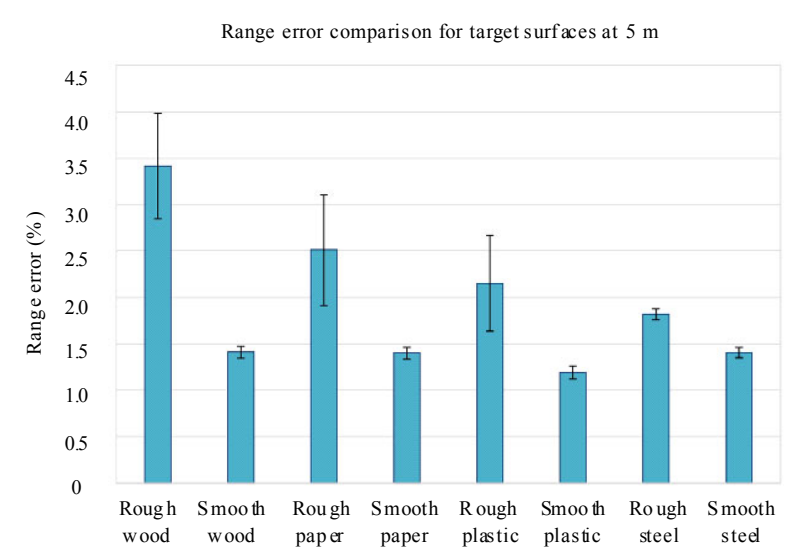

Fig. 6 Comparison of range error for target surfaces with different reflectivity and roughness. 


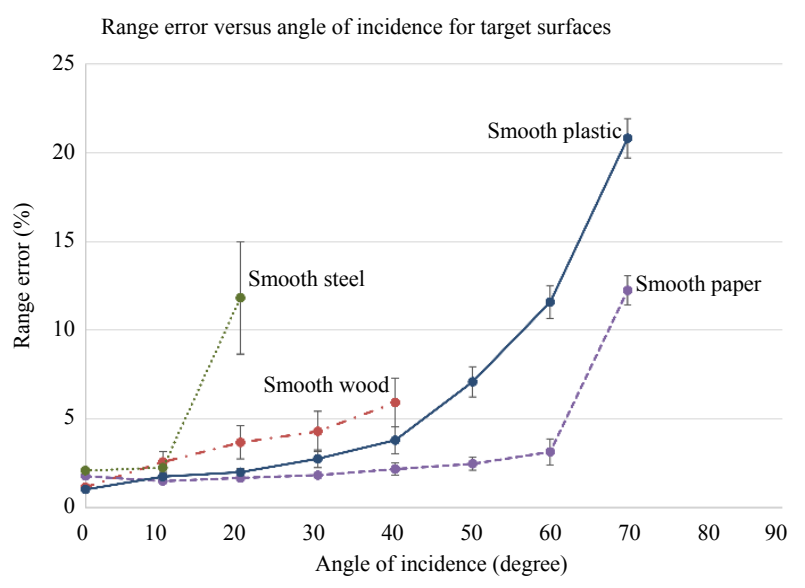

Fig. 7 Comparison of range error versus angle of incidence for target surfaces with different reflectivity.

\subsection{Acquisition time step}

From (12) and (14), it can be seen that the reflected intensity is inversely proportional to the time step used to acquire a series of image slices and error in the calculated range $\delta_{r}$ shows direct dependency on the time step parameter. Under the same setup condition where all parameters are regarded as constants, range error is expected to increase with time step value in theory. Figure 8 shows the range error trend analyzed based on average of 30 measurements. This set of experimental result clearly points out that a smaller time step should be selected to gain higher accuracy. However, the choice of the time step used is a trade-off between range accuracy and processing cost in terms of time and effort which should be taken into consideration.

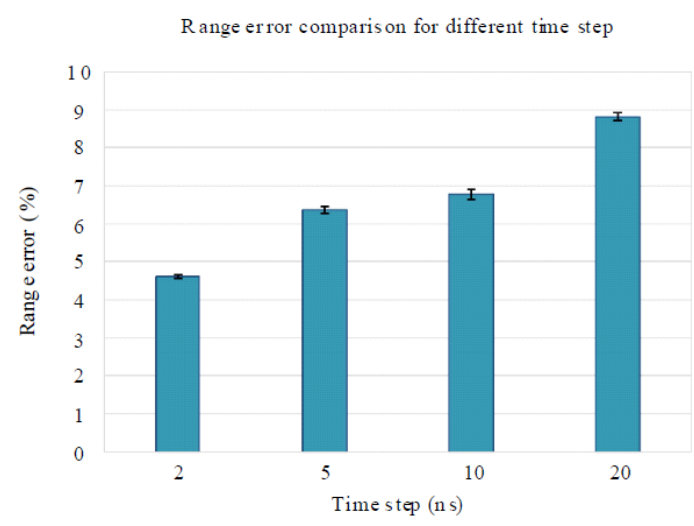

Fig. 8 Comparison of range error for different time steps.

\section{Conclusions}

In summary, this paper has demonstrated the influence of multiple factors on range gated reconstruction through theoretical and experimental investigation. Based on the operating principle of time slicing technique, LADAR, and BRDF, theoretical derivation of range gated reconstruction model is presented. Range accuracy shows dependency on the SNR which is proportional to the reflected laser intensity when the system noise level remains unchanged.

Impact on the accuracy of range reconstruction is studied from the perspective of distance, target reflection, and acquisition time step. Each influence factor is analyzed theoretically, and experimental investigation is performed to validate the theoretical discussion. It is concluded that our experimental results agree well with the theoretical analysis where the expected range error trends are shown.

The presented findings establish a comprehensive understanding of multiple influence factors which may benefit various applications and serve as references to perform correction or compensation. In future, follow-up improvement of range reconstruction can be proposed and additional effects caused by illumination, sensor, and noise can be included.

\section{Acknowledgment}

The authors gratefully acknowledge the support of funding from Ministry of Higher Education, Malaysia under the Grant No: FRGS/1/2016/STG02/ MUSM/02/1.

Open Access This article is distributed under the terms of the Creative Commons Attribution 4.0 International License (http://creativecommons.org/licenses/by/4.0/), which permits unrestricted use, distribution, and reproduction in any medium, provided you give appropriate credit to the original author(s) and the source, provide a link to the Creative Commons license, and indicate if changes were made. 


\section{References}

[1] P. Huke, R. Klattenhoff, and C. V. Kopylow, "Novel trends in optical non-destructive testing methods," Journal of the European Optical Society Rapid Publications, 2013, 8(13): 1-7.

[2] M. C. Amann, T. Bosch, M. Lescure, R. Myllylä, and M. Rioux, "Laser ranging: a critical review of usual techniques for distance measurement," Optical Engineering, 2001, 40(1): 10-19.

[3] Y. Liu, W. Zhang, T. Xu, J. He, F. Zhang, and F. Li, "Fiber laser sensing system and its applications," Photonic Sensors, 2011, 1(1): 43-53.

[4] A. Velten, T. Willwacher, O. Gupta, A. Veeraraghavan, M. G. Bawendi, and R. Raskar, "Recovering three-dimensional shape around a corner using ultrafast time-of-flight imaging," Nature Communications, 2012, 3(745): 1-8.

[5] D. Monnin, A. L. Schneider, F. Christnacher, and Y. Lutz, "A 3D outdoor scene scanner based on a night-vision range-gated active imaging system," in Third International Symposium on 3D Data Processing, Visualization, and Transmission, Chapel Hill, NC, pp. 938-945, 2006.

[6] X. W. Wang, Y. Zhou, S. T. Fan, J. He, and Y. L. Liu, "Range-gated laser stroboscopic imaging for night remote surveillance," Chinese Physics Letters, 2010, 27(9): 94-97.

[7] J. Busck, "Underwater 3-D optical imaging with a gated viewing laser radar," Optical Engineering, 2005, 44(11): 6456-6468.

[8] C. Tan, G. Seet, A. Sluzek, and D. He, "A novel application of range-gated underwater laser imaging system (ULIS) in near-target turbid medium," Optical and Lasers Engineering, 2005, 43(9): 995-1009.

[9] J. Busck and H. Heiselberg, "Gated viewing and high-accuracy three-dimensional laser radar," Applied Optics, 2004, 43(24): 4705-4710.

[10] S. Y. Chua, X. Wang, N. Guo, and C. S. Tan, "Range compensation for accurate 3D imaging system," Applied Optics, 2016, 55(1): 153-158.

[11] B. Höfle and N. Pfeifer, "Correction of laser scanning intensity data: Data and model-driven approaches," ISPRS Journal of Photogrammetry and Remote Sensing, 2007, 62(6): 415-433.

[12] X. W. Wang, Y. F. Liu, and Y. Zhou, "Triangularrange-intensity profile spatial-correlation method for 3D super-resolution range-gated imaging," Applied Optics, 2013, 52(30): 7399-7406.

[13] S. Y. Chua, X. Wang, N. Guo, C. S. Tan, T. Y. Chai, and G. G. L. Seet, "Improving three-dimensional (3D) range gated reconstruction through time-of-flight (TOF) imaging analysis," Journal of European Optical Society Rapid Publications, 2016, 11: 16015-1-16015-6.

[14] B. Fu, K. Yang, J. Rao, and M. Xia, "Analysis of MCP gain selection for underwater range-gated imaging applications based on ICCD," Journal of Modern Optics, 2010, 57(5): 408-417.

[15] X. Wang, L. Hu, Q. Zhi, Z. Chen, and W. Jin, "Influence of range-gated intensifiers on underwater imaging system SNR," Proc. SPIE, 2013, 8912: 89120E.

[16] M. Laurenzis, F. Christnacher, D. Monnin, and T. Scholz, "Investigation of range-gated imaging in scattering environments," Optical Engineering, 2012, 51(6): 061303.

[17] R. D. Richmond and S. C. Cain, Direct-detection LADAR systems. U. S. A.: SPIE Press, 2010: 1-26.

[18] O. Steinvall, "Effects of target shape and reflection on laser radar cross sections," Applied Optics, 2000, 39(24): 4381-4391.

[19] D. Kong, J. Chang, P. Gong, Y. Liu, B. Sun, X. Liu, et al., "Analysis and improvement of SNR in FBG sensing system," Photonic Sensors, 2012, 2(2): 148-157.

[20] S. S. Patil and A. D. Shaligram, "On-line defect detection of aluminum coating using fiber optic sensor," Photonic Sensors, 2015, 5(1): 72-78.

[21] S. Y. Chua, X. Wang, N. Guo, C. S. Tan, and T. Y. Chai, "Effects of target reflectivity on the reflected laser pulse for range estimation," in Progress In Electromagnetics Research Symposium Proceedings, Prague, Czech Republic, pp. 2695-2699, 2015. 